\title{
Attenuation correction for a high-resolution polarimetric X-band weather radar
}

\section{T. Otto and H. W. J. Russchenberg}

Remote Sensing of the Environment, International Research Centre for Telecommunications and Radar (IRCTR), Department of Electrical Engineering, Mathematics and Computer Science, Delft University of Technology, Delft, The Netherlands

\begin{abstract}
In 2007, IRCTR (Delft University of Technology) installed a new polarimetric X-band LFMCW radar (IDRA) at the meteorological observation site of Cabauw, The Netherlands. It provides plan position indicators (PPI) at a fixed elevation with a high range resolution of either $3 \mathrm{~m}$ or $30 \mathrm{~m}$ at a maximum observation range of $1.5 \mathrm{~km}$ and $15 \mathrm{~km}$, respectively. IDRA aims to monitor precipitation events for the long-term analysis of the hydrological cycle. Due to the specifications of IDRA, the spatial and temporal variability of a large range of rainfall intensities (from drizzle to heavy convective rain) can be studied.

Even though the usual observation range of IDRA is limited to $15 \mathrm{~km}$, attenuation due to precipitation can be large enough to seriously affect the measurements. In this contribution we evaluate the application of a combined method to correct for the specific and the differential attenuation, and in the same vein estimate the parameters of the raindrop-size distribution. The estimated attenuations are compared to a phase constraint attenuation correction method.
\end{abstract}

\section{Introduction}

Weather radars are a widely used measurement device to observe the spatial distribution and the temporal evolution of precipitation. In Europe over 150 operational C-band weather radars are employed by the meteorological services ${ }^{1}$. Nowadays, the operational weather radar networks are complemented by compact $\mathrm{X}$-band systems as gap fillers, McLaughlin et al. (2009). However, the attenuation of microwaves by precipitation is stronger at X-band than at C-

\section{Correspondence to: $\mathrm{T}$. Otto}

(t.otto@tudelft.nl)

${ }^{1}$ OPERA - Operational programme for the exchange of weather radar information, http://www.knmi.nl/opera band. Radar measurements must be corrected for attenuation before they are used for a quantitative determination of precipitation parameters. A first formulation of attenuation correction for weather radar measurements was provided by Hitschfeld and Bordan (1954). However, without constraining the path-integrated attenuation (PIA) by independent measurements, e.g. rain gauges or microwave links, the attenuation correction is unstable. Polarimetric weather radars operating at linear horizontal/vertical polarisation basis have the capability to measure the differential propagation phase that is a forward scattering effect like attenuation. Scattering computations show that the differential propagation phase is linearly related to the attenuation, Holt (1988), and can those be used to provide an estimate of the PIA. However, the relation between the differential propagation phase and the attenuation depends on the microphysics of the precipitation, Bringi et al. (1990). Testud et al. (2000) introduced an algorithm that uses the differential propagation phase to estimate the PIA that is subsequently distributed among the range bins weighted by the reflectivity. Additionally, self-consistency among the weather radar observables involved in the attenuation correction may be imposed in order to account for the variability of the relation among the differential propagation phase and the attenuation, Bringi et al. (2001), Gorgucci and Baldini (2007).

Attenuation correction is also a prerequisite for IDRA, the polarimetric, horizontally scanning X-band weather radar of IRCTR installed at the meteorological observatory at Cabauw $^{2}$, The Netherlands. One purpose of IDRA is the long-term monitoring of microphysics of precipitation. The method proposed in Chandra et al. (1990) is able to jointly perform the attenuation correction and the raindrop-size distribution retrieval for rain. It was successfully applied to Cband weather radar data. The purpose of this contribution is the evaluation of this method for X-band weather radar data

\footnotetext{
${ }^{2}$ http://www.cesar-database.nl
} 

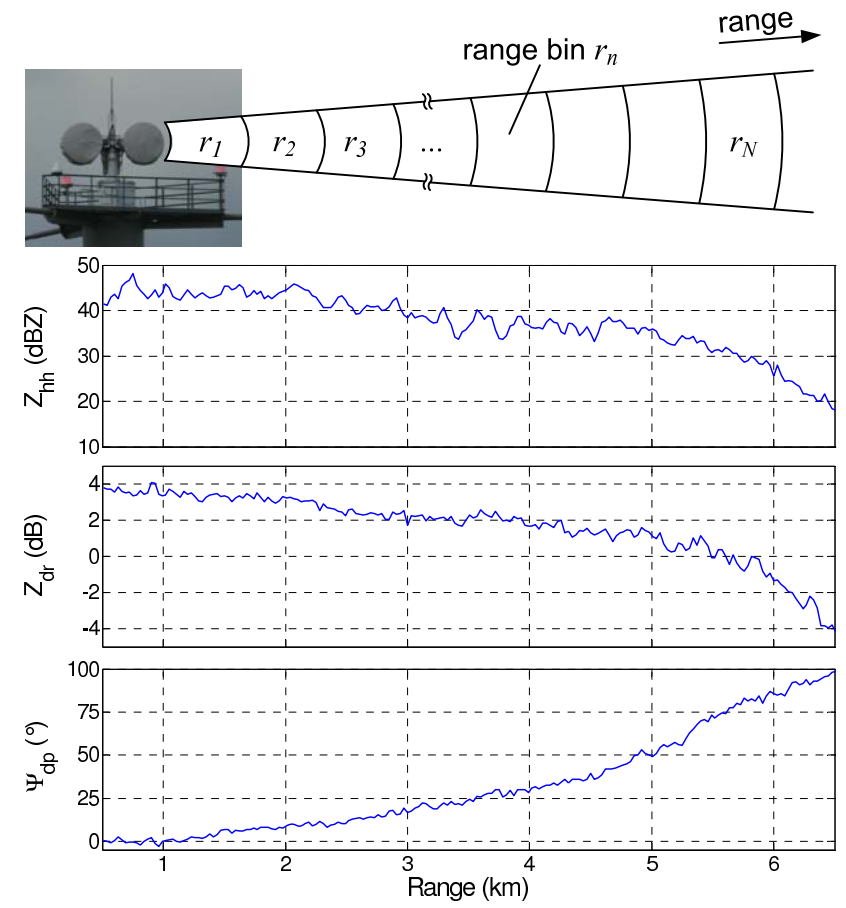

Fig. 1. The radar geometry is shown in the upper part of this figure. The lower part shows rayplots of an IDRA measurement (26 May $2009,02: 00$ UTC) with heavy precipitation leading to a very strong specific and differential attenuation that deteriorate the measured reflectivity $Z_{\mathrm{hh} \text {, att }}$ and the differential reflectivity $Z_{\mathrm{dr} \text {,att }}$ (upper two ray plots). The measured differential propagation phase shows the expected range-cumulative behaviour (ray plot at the bottom).

by applying it to IDRA data. Therefore, the attenuations derived by this method are compared to the phase constraint attenuation correction method with imposed self-consistency, Gorgucci and Baldini (2007).

\section{Basics}

The natural variability of rainfall can be described by the three-parameter raindrop-size distribution (RDSD) that normalised with respect to the liquid water content can be written as, Bringi and Chandrasekar (2001),

$N(D)=N_{w} f(\mu)\left(\frac{D}{D_{0}}\right)^{\mu} \mathrm{e}^{-(3.67+\mu) \frac{D}{D_{0}}}$

with

$f(\mu)=\frac{6}{3.67^{4}} \frac{(3.67+\mu)^{\mu+4}}{\Gamma(\mu+4)}$

where $D$ is the equivolumetric raindrop diameter, $D_{0}$ is the median volume diameter, $N_{w}$ is the concentration, and $\mu$ is the shape parameter.

The measurements of an homogeneous ensemble of raindrops by polarimetric weather radars can be expressed in terms of the RDSD, and the scattering amplitudes of the raindrops as follows:

$$
\begin{aligned}
& Z_{\mathrm{hh}}=10 \log _{10}\left(\frac{\lambda^{4}}{\pi^{5}|K|^{2}} \int_{D} \sigma_{\mathrm{hh}}(D) N(D) d D\right) \\
& Z_{\mathrm{dr}}=10 \log _{10}\left(\frac{\int_{D} \sigma_{\mathrm{hh}}(D)\left(\frac{D}{D_{0}}\right)^{\mu} \mathrm{e}^{-(3.67+\mu) \frac{D}{D_{0}}} d D}{\int_{D} \sigma_{\mathrm{vv}}(D)\left(\frac{D}{D_{0}}\right)^{\mu} \mathrm{e}^{-(3.67+\mu) \frac{D}{D_{0}}} d D}\right) \\
& K_{\mathrm{dp}}^{1-\text { way }}=\frac{180}{\pi} \lambda \int_{D} \Re\left[f_{\mathrm{hh}}(D)-f_{\mathrm{vv}}(D)\right] N(D) d D .
\end{aligned}
$$

The intrinsic weather radar observables in Eqs. (3) to (5) represent the co-polarised reflectivity at horizontal polarisation $Z_{\mathrm{hh}}(\mathrm{dBZ})$, the differential reflectivity $Z_{\mathrm{dr}}(\mathrm{dB})$, and the specific differential phase $K_{\mathrm{dp}}^{1-\text { way }}(\% / \mathrm{km})$ which is the range derivative of the differential propagation phase $\Psi_{\mathrm{dp}}$. Furthermore, $\lambda$ is the wavelength, $|K|^{2}$ is a dielectric factor, $\sigma_{\mathrm{hh} / \mathrm{vv}}$ are the radar cross sections, and $f_{\mathrm{hh} / \mathrm{vv}}$ are the forward scattering amplitudes of the raindrops at linear horizontal and linear vertical polarisations. For the purpose of this contribution, numerical values of the radar cross sections and the forward scattering amplitudes of raindrops have been computed for a frequency of $9.475 \mathrm{GHz}$ (IDRA) by the Fredholm Integral Method, Holt et al. (1978), assuming dielectric spheroids with the axis ratio given by Illingworth et al. (2000), and the complex permittivity of water at a temperature of $10^{\circ} \mathrm{C}$, Liebe et al. (1991).

The propagation of electromagnetic waves through rain leads to attenuation of the measured reflectivity and the differential reflectivity, Fig. 1:

$Z_{\mathrm{hh}, \text { att }}\left(r_{n}\right)=Z_{\mathrm{hh}}\left(r_{n}\right)-2 \int_{r_{1}}^{r_{n-1}} \alpha_{\mathrm{hh}}^{1-\text { way }}(r) d r$
$Z_{\mathrm{dr}, \text { att }}\left(r_{n}\right)=Z_{\mathrm{dr}}\left(r_{n}\right)-2 \int_{r_{1}}^{r_{n-1}} \alpha_{\mathrm{h}-\mathrm{v}}^{1-\text { way }}(r) d r$.

The specific attenuation $\alpha_{\mathrm{hh}}^{1-\text { way }}(\mathrm{dB} / \mathrm{km})$ and the differential attenuation $\alpha_{\mathrm{h}-\mathrm{v}}^{1-\text { way }}(\mathrm{dB} / \mathrm{km})$ in the equations above are given by

$\alpha_{\mathrm{hh}}^{1-\text { way }}=8.686 \lambda \int_{D} \Im\left[f_{\mathrm{hh}}(D)\right] N(D) d D$

and

$\alpha_{\mathrm{h}-\mathrm{v}}^{1-\text { way }}=8.686 \lambda \int_{D} \Im\left[f_{\mathrm{hh}}(D)-f_{\mathrm{vv}}(D)\right] N(D) d D$.

A method to correct polarimetric weather radar measurements for attenuation has been introduced by Chandra et al. (1990). It is based on the estimation of the RDSD parameters that are subsequently used to determine the corresponding attenuations by scattering computations. The block diagram of this method, shown in Fig. 2, is explained next. 


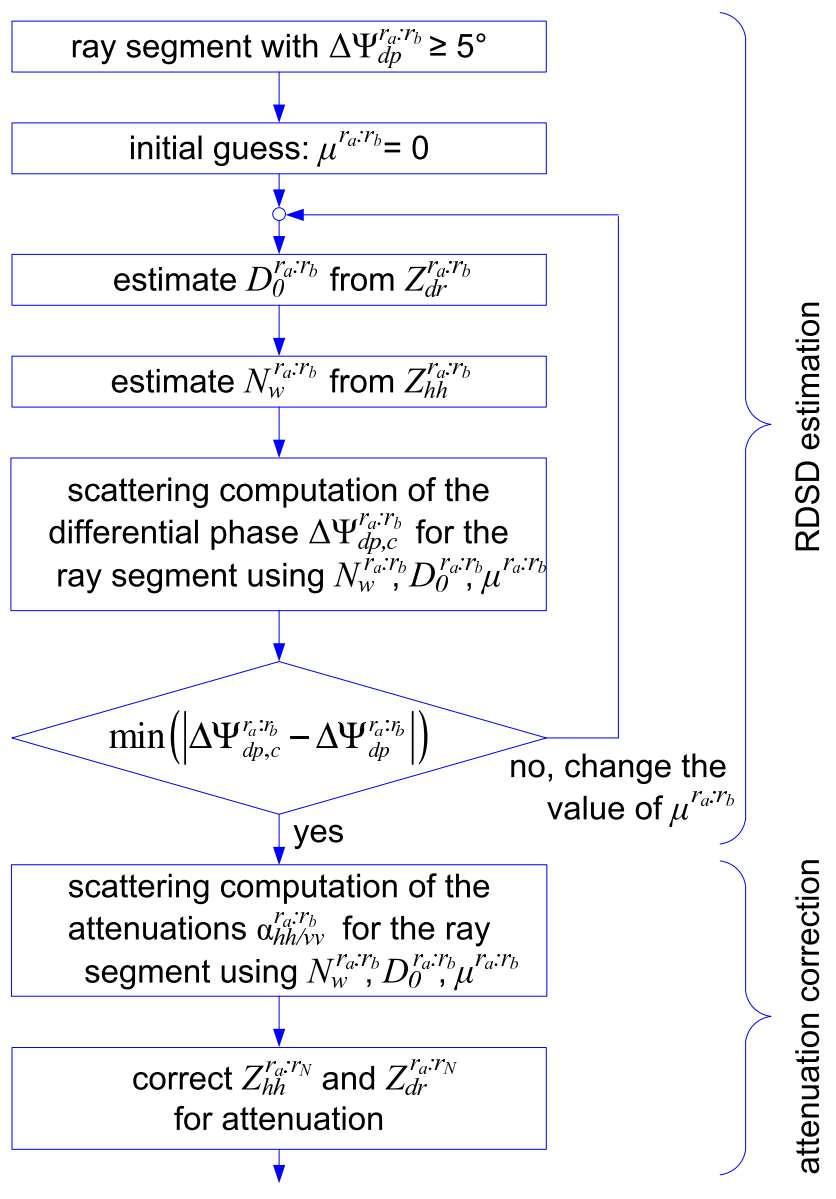

go to the next ray segment

Fig. 2. Block diagram of the method of Chandra et al. (1990) to extract the RDSD parameters, and, subsequently, to correct for attenuation.

A measured ray of the integrated weather radar observables $Z_{\mathrm{hh}, \text { att }}, Z_{\mathrm{dr} \text {,att }}$ and $\Psi_{\mathrm{dp}}$, such as the one exemplary shown in Fig. 1, is divided into ray segments satisfying $\Delta \Psi_{\mathrm{dp}}^{r_{a}: r_{b}}=$ $\Psi_{\mathrm{dp}}\left(r_{b}\right)-\Psi_{\mathrm{dp}}\left(r_{a}\right) \geq 5^{\circ}$. A precondition for the application of this method is a given raindrop shape and a temperature to calculate the complex permittivity of water. Starting at close range to the radar, i.e. $r_{a}=r_{1}$, Fig. 1, the median volume diameter $D_{0}^{r_{a}: r_{b}}$ is estimated via pre-calculated look-up tables from the measured differential reflectivity $Z_{\mathrm{dr} \text { att }}^{r_{a}: r_{b}}$, assuming an initial shape parameter $\mu^{r_{a}: r_{b}}=0$ (blue curve in Fig. 3). After determining $N_{w}^{r_{a}: r_{b}}$ again via pre-calculated look-up tables (red curve in Fig. 3), scattering computations with the determined RDSD parameters are carried out in order to compute the expected differential propagation phase $\Delta \Psi_{\mathrm{dp}, \mathrm{c}}^{r_{a}: r_{b}}$ for the ray segment. The optimum shape parameter $\mu^{r_{a}: r_{b}}$ for the ray segment is found by minimising iteratively the difference among the measured and the computed differential propagation phase of the ray segment, i.e. $\min \left(\left|\Delta \Psi_{\mathrm{dp}, \mathrm{c}}^{r_{a}: r_{b}}-\Delta \Psi_{\mathrm{dp}}^{r_{a}: r_{b}}\right|\right)$. The range of possible shape pa-

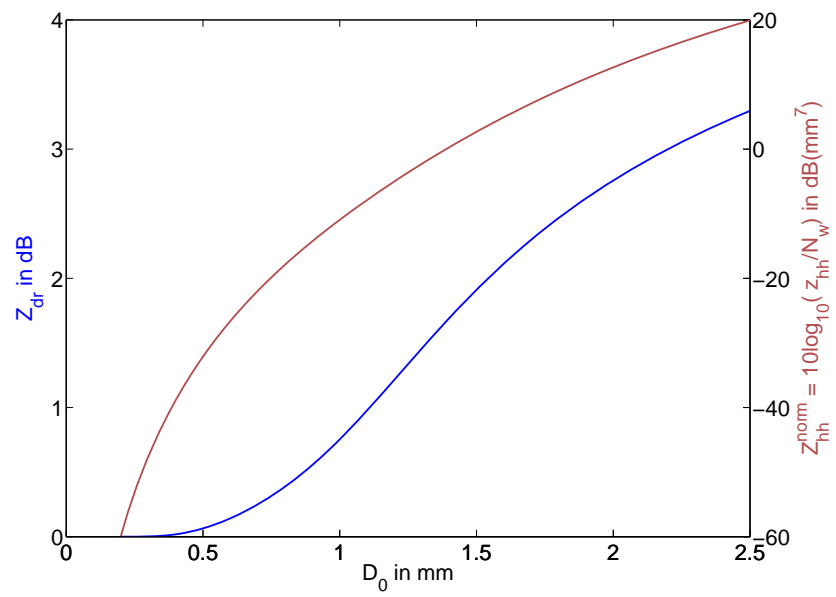

Fig. 3. Results of scattering computations to relate the differential reflectivity (blue curve) and the normalised reflectivity at horizontal polarisation (red curve) to the median volume diameter. The shape parameter is $\mu=0$, and the raindrop axis ratio of Illingworth et al. (2000) was used.

rameters was limited within $-2 \leq \mu^{r_{a}: r_{b}} \leq 10$. Once the RDSD is characterised in this way, scattering computations are carried out again in order to estimate the attenuations, Eqs. (8) and (9), for the ray segment. After correcting $Z_{\mathrm{hh}, \text { att }}^{r_{a}: r_{N}}$ and $Z_{\mathrm{dr}, \text { att }}^{r_{a}: r_{N}}$ for the attenuation, the method is applied to the adjacent ray segment, i.e. $r_{a}=r_{b+1}$.

This method is only suited for rain, i.e. it is likely to fail in the presence of ground clutter or other hydrometeors such as graupel, hail or snow. Furthermore, Otto et al. (2009) showed that the application of this method is limited for IDRA to $0.5 \mathrm{~dB} \leq Z_{\mathrm{dr}} \leq 2.7 \mathrm{~dB}$ in order to estimate the median volume diameter within $\pm 0.15 \mathrm{~mm}$. Thus, the method is not suited for light rain (rain rates $<3 \mathrm{~mm} / \mathrm{h}$ ) as well as for strong rain (rain rates $>25 \mathrm{~mm} / \mathrm{h}$ ). In the case of strong rain, scattering computations show that the attenuation at X-band can be alternatively estimated by

$\alpha_{\mathrm{hh}}(\mathrm{dB} / \mathrm{km})=0.25 K_{\mathrm{dp}}\left({ }^{\circ} / \mathrm{km}\right)$

and

$\alpha_{\mathrm{h}-\mathrm{v}}(\mathrm{dB} / \mathrm{km})=0.05 K_{\mathrm{dp}}\left({ }^{\circ} / \mathrm{km}\right)$.

However, Eqs. (10) and (11) introduce uncertainties due to the variability of the microphysics of precipitation, Bringi et al. (1990), and due to the estimation of $K_{\mathrm{dp}}$, Georgiou et al. (2009).

For each ray segment the RDSD is estimated from attenuated reflectivity and differential reflectivity measurements which means that the median volume diameter will be underestimated, blue curve in Fig. 3. This in turn results in an underestimation of $Z_{\mathrm{hh}}^{\mathrm{norm}}$, red curve in Fig. 3, and an overestimation of the concentration since $N_{w}=$ $10^{\left(Z_{\mathrm{hh}, \text { att }}-Z_{\mathrm{hh}}^{\text {norm }}\right) / 10}\left(\mathrm{~mm}^{-1} \mathrm{~m}^{-3}\right)$. At $\mathrm{X}$-band this may yield for $\mu=0$ to an underestimation of $0.3 \mathrm{~mm}$ for $D_{0}$, and an 


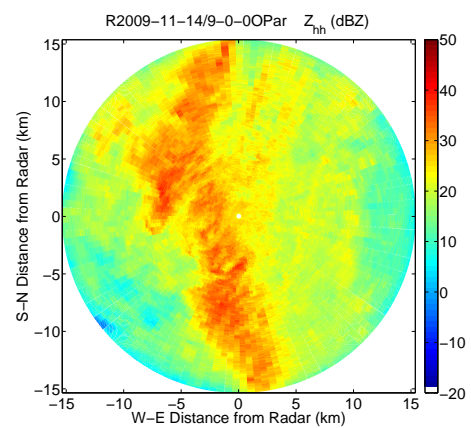

(a) $Z_{h h, a t t}(\mathrm{dBZ})$

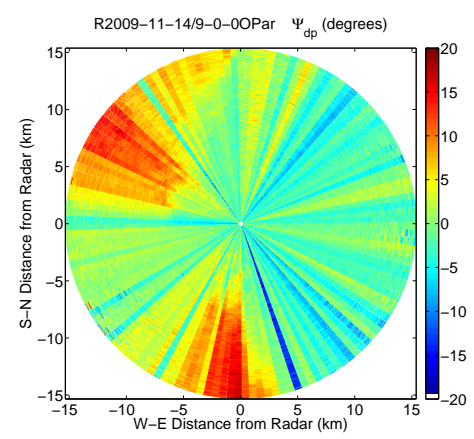

(d) $\Psi_{d p}\left({ }^{\circ}\right)$

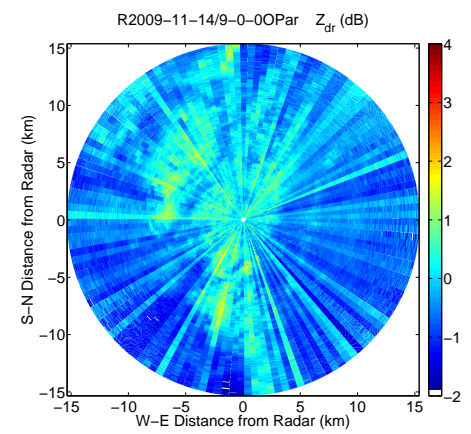

(b) $Z_{d r, a t t}(\mathrm{~dB})$

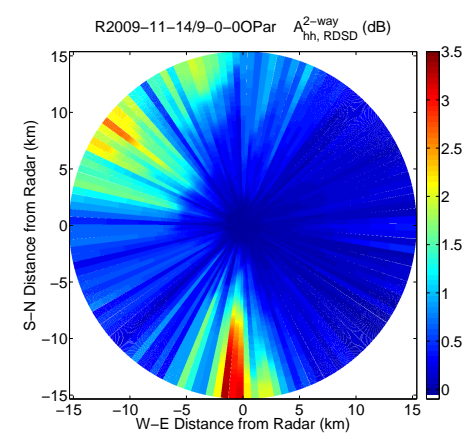

(e) $A_{h h, R D S D}^{2-w a y}(\mathrm{~dB})$

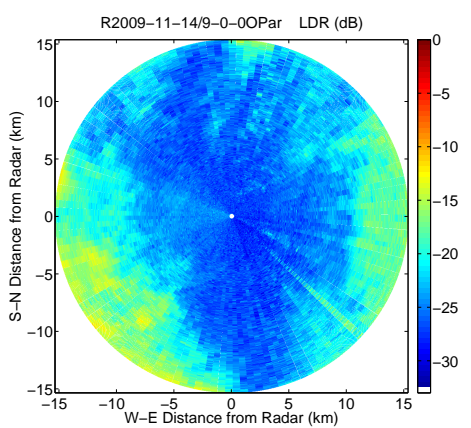

(c) $L D R(\mathrm{~dB})$

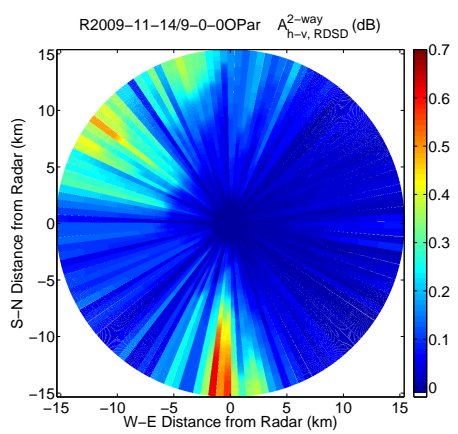

(f) $A_{h-v, R D S D}^{2-w a y}(\mathrm{~dB})$

Fig. 4. IDRA precipitation measurements from the 14 November 2009 at 09:00 UTC. Shown are PPI's of (a) the reflectivity $Z_{\mathrm{hh}, \text { att }}(\mathrm{dBZ}$ ), (b) the differential reflectivity $Z_{\mathrm{dr} \text {, att }}(\mathrm{dB})$, (c) the linear depolarisation ratio LDR $(\mathrm{dB})$ and $(\mathbf{d})$ the differential propagation phase $\Psi_{\mathrm{dp}}\left({ }^{\circ}\right)$. (e) and (f) show the 2-way cumulative attenuation at horizontal polarisation and the differential attenuation, respectively, derived by the method of Chandra et al. (1990).

overestimation of $N_{w}$ up to a factor of 100. For the computation of the attenuations, Eqs. (8) and (9), this may yield in turn to an over- or underestimation. In any case, the RDSD parameters are estimated again after the first run of this method from the attenuation corrected reflectivities. The presented method is applied in the next section to IDRA measurements in order to assess the accuracy of the estimated attenuations. Therefore, they are compared to the attenuations derived by the phase constraint method with imposed self-consistency of Gorgucci and Baldini (2007).

\section{Application to IDRA data}

Figure 4 shows a precipitation measurement of IDRA from the 14 November 2009, 09:00 UTC. IDRA is located in the centre of these plan-position indicators (PPI), that from Fig. 4a to dillustrate the measured weather radar observables reflectivity $Z_{\mathrm{hh} \text {, att }}(\mathrm{dBZ})$, differential reflectivity $Z_{\mathrm{dr} \text {,att }}(\mathrm{dB})$, linear depolarisation ratio LDR $(\mathrm{dB})$, and differential propagation phase $\Psi_{\mathrm{dp}}\left({ }^{\circ}\right)$. The presence of propagation effects is not evident in the reflectivity and the differential reflectivity measurement. However, the measured differential phase,
Fig. $4 \mathrm{~d}$, shows cumulative values of up to $20^{\circ}$ over the range of $15 \mathrm{~km}$ in some sectors.

Using the measured $Z_{\mathrm{hh} \text {,att }}, Z_{\mathrm{dr} \text {,att }}$ and $\Psi_{\mathrm{dp}}$, the attenuations were determined by the method of Chandra et al. (1990) introduced in the preceding section. The resulting cumulative attenuation at horizontal polarisation and the differential attenuation are shown in Fig. $4 \mathrm{e}$ and $\mathrm{f}$.

For a comparison, the attenuations were also derived by the method outlined in Gorgucci and Baldini (2007). This method relies on a first estimation of the attenuations by the application of the phase constraint method of Testud et al. (2000). Then the self-consistency equations

$\alpha_{\mathrm{hh}}=a_{1} z_{\mathrm{hh}}^{b_{1}} z_{\mathrm{dr}}^{c_{1}} K_{\mathrm{dp}}^{d_{1}}$

and

$\alpha_{\mathrm{h}-\mathrm{v}}=a_{2} z_{\mathrm{hh}}^{b_{2}} z_{\mathrm{dr}}^{c_{2}} K_{\mathrm{dp}}^{d_{2}}$

are imposed as outlined in Gorgucci and Baldini (2007), resulting in an adjustment of the estimated attenuations. The reflectivities $z_{\mathrm{hh}}$ and $z_{\mathrm{dr}}$ in Eqs. (12) and (13) are in linear units, and the coefficients of these self-consistency equations for the frequency of IDRA can be found in Table 1. In the following comparison of the attenuations, the subscript SCPC 

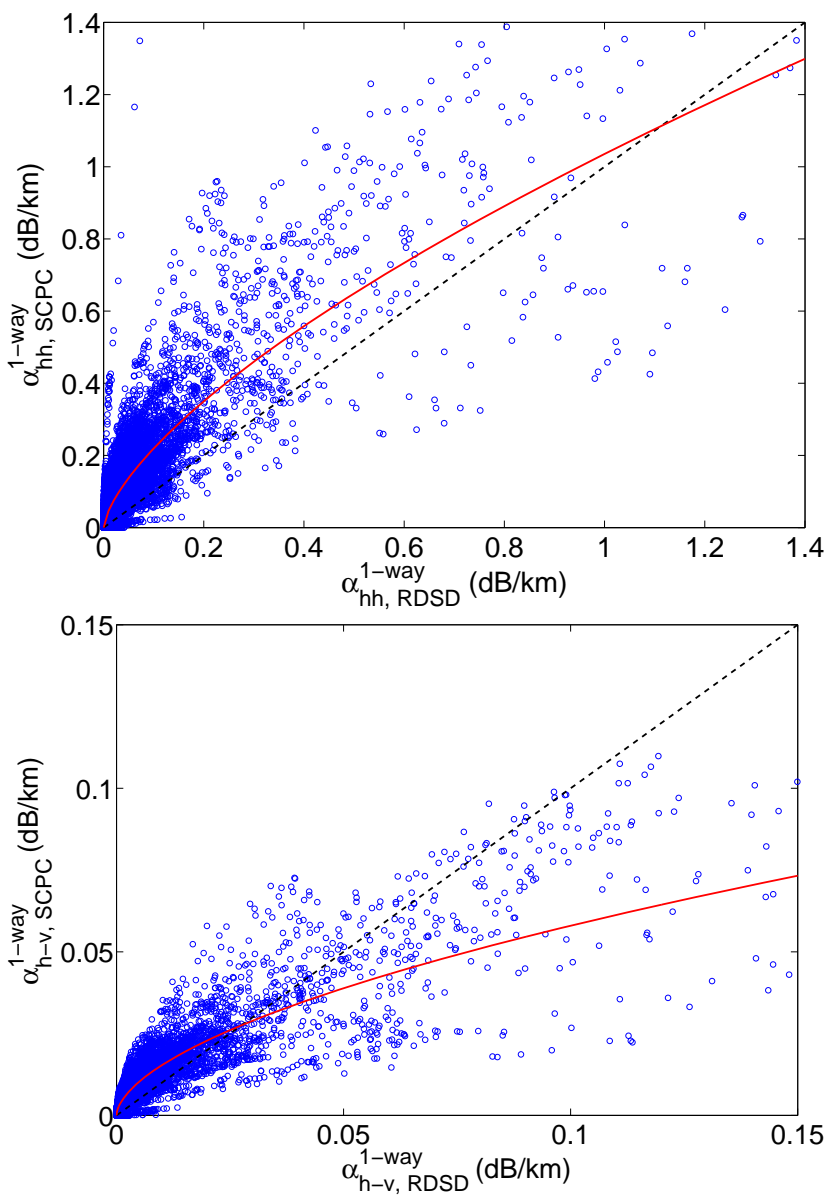

Fig. 5. Scatter plots comparing (a) the specific attenuations at horizontal polarisation $\alpha_{\mathrm{hh}}^{1-\text { way }}$, and (b) the differential attenuations $\alpha_{\mathrm{h}-\mathrm{v}}^{1-\text { way }}$ estimated by the methods of Chandra et al. (1990) (RDSD) and Gorgucci and Baldini (2007) (SCPC) for 35317 range bins of two PPI's measured by IDRA. The dashed black line corresponds to $x=y$. The red line is a power-law fit.

denotes attenuations derived by the self-consistency method of Gorgucci and Baldini (2007), and the subscript RDSD denotes attenuations derived by the method of Chandra et al. (1990).

Figure 5 shows two scatter plots comparing the attenuations $\alpha_{\mathrm{hh}}^{1-\text { way }}$ and $\alpha_{\mathrm{h}-\mathrm{v}}^{1-\text { way }}$ estimated by the two methods presented above from IDRA data. The data comprise 35317 range bins from two PPI's measured the 25 May 2009, 20:23 UTC, and the 14 November 2009, 09:00 UTC, Fig. 4. The red lines in the scatter plots are power law fits $y=m x^{n}$ to the data with $m_{\mathrm{hh}}=1, n_{\mathrm{hh}}=0.67$, and $m_{\mathrm{h}-\mathrm{v}}=0.22$, $n_{\mathrm{h}-\mathrm{v}}=0.58$. The general tendency shows that for small attenuations $\alpha_{\mathrm{hh}}^{1-\text { way }}<1.1 \mathrm{~dB} / \mathrm{km}$ and $\alpha_{\mathrm{h}-\mathrm{v}}^{1-\text { way }}<0.03 \mathrm{~dB} / \mathrm{km}$, the RDSD method is underestimating the attenuations compared to SCPC method, whereas larger attenuations are overestimated. This non-linear correlation between the estimated attenuations that is most notably in Fig. $5 \mathrm{~b}$ may be attributed to the uncertainties introduced by estimating the RDSD pa-
Table 1. Coefficients for the self-consistency Eqs. (12) and (13) derived for a frequency of $9.475 \mathrm{GHz}$ for a set of 1500 normalised raindrop-size distributions with varying $N_{w}, D_{0}, \mu$, raindrop axis ratios and temperatures.

\begin{tabular}{cccc}
\hline$a_{1}$ & $b_{1}$ & $c_{1}$ & $d_{1}$ \\
$4.95 \times 10^{-3}$ & 0.463 & -1.321 & 0.539 \\
\hline$a_{2}$ & $b_{2}$ & $c_{2}$ & $d_{2}$ \\
$7.58 \times 10^{-4}$ & 0.378 & 0.416 & 0.64 \\
\hline
\end{tabular}

rameters from attenuated reflectivities. Depending on the actual values of the reflectivities, the resulting opposite errors of $D_{0}$ and $N_{w}$ do show non-linearities, Fig. 3. One may think of additional iterations within the RDSD method to improve the estimated attenuations. However, this would yield an increase of the processing time. Instead, at X-band it is suggested to perform the attenuation correction prior to the estimation of the RDSD parameters.

\section{Conclusions}

The purpose of this contribution was the evaluation of a method to estimate the RDSD parameters and in the same vein to perform a correction for attenuation for polarimetric weather radar data at X-band, Chandra et al. (1990). The focus was laid on the estimated attenuations by comparing them to a phase-constraint attenuation correction method with imposed self-consistency, Gorgucci and Baldini (2007). Small attenuations were underestimated by the RDSD method compared to the SCPC method whereas large attenuations were overestimated by the RDSD method. The attenuations estimated by the RDSD method result from scattering computations with RDSD parameters that are estimated from attenuated reflectivities. This may result in an underestimation of the median volume diameter $D_{0}$ but an overestimation of the concentration $N_{w}$ that may explain the observed deviations of the estimated attenuations. Since the RDSD method is only applicable for a limited differential reflectivity range, no attenuation estimate is provided for weak and for strong rain. For X-band, this suggests to apply the attenuation correction and the RDSD estimation separately, i.e. first correct the reflectivities for attenuation using a phaseconstraint method, followed by the estimation of the RDSD parameters. However it must be stressed, that the results of the comparison of the attenuation correction methods in this contribution can not be regarded as absolute. As a next step, we shall take advantage of the operational C-band weather radar of the Royal Netherlands Meteorological Institute at De Bilt that is situated in the vicinity of IDRA in a distance of approximately $25 \mathrm{~km}$. The analysis and comparison of simultaneous measurements of precipitation events shall enable us to estimate the performance of attenuation correction methods. 


\section{References}

Bringi, V. N. and Chandrasekar, V.: Polarimetric Doppler Weather Radar, p. 410, Cambridge University Press, 1st edn., 2001.

Bringi, V. N., Chandrasekar, V., Balakrishnan, N., and Zrnić, D. S.: An Examination of Propagation Effects in Rainfall on Radar Measurements at Microwave Frequencies, J. Atmos. Oceanic Technol., 7, 829-840, 1990.

Bringi, V. N., Keenan, T. D., and Chandrasekar, V.: Correcting CBand Radar Reflectivity and Differential Reflectivity Data for Rain Attenuation: A Self-Consistent Method With Constraints, IEEE Trans. Geosci. Remote Sens., 39, 1906-1915, 2001.

Chandra, M., Schroth, A., and Lüneburg, E.: The Influence of Raindropsize Distribution on the Determination of Microwave Propagation Properties by Polarimetric Radars, in: Geoscience and Remote Sensing Symposium, IGARSS 90, 1041-1045, 1990.

Georgiou, S., Figueras i Ventura, J., and Unal, C. M. H.: Estimation of differential and specific differential phase with the IDRA $\mathrm{X}$-band Doppler polarimetric radar for improving rain rate retrieval, in: Proceedings of the 8th International Symposium of tropospheric profiling, Delft, The Netherlands, ISBN 978-906960-233-2, edited by: Apituley, A., Russchenberg, H. W. J., and Monna, W. A. A., 2009.

Gorgucci, E. and Baldini, L.: Attenuation and Differential Attenuation Correction of C-Band Radar Observations Using a Fully Self-Consistent Methodology, IEEE Geosci. Remote Sens. Lett., 4, 326-330, 2007.
Hitschfeld, W. and Bordan, J.: Errors inherent in the radar measurement of rainfall at attenuating wavelengths, J. Meteorol., 11, 58-67, 1954.

Holt, A. R.: Extraction of differential propagation phase from data from S-band circularly polarized radars, Electron. Lett., 24, 1241-1244, 1988.

Holt, A. R., Uzunoglu, N. K., and Evans, B. G.: An Integral Equation Solution to the Scattering of Electromagnetic Radiation by Dielectric Spheroids and Ellipsoids, IEEE Trans. Antennas Propag., AP-26, 706-712, 1978.

Illingworth, A. J., Blackman, T. M., and Goddard, J. W. F.: Improved rainfall estimates in convective storms using polarisation diversity radar, Hydrol. Earth Syst. Sci., 4, 555-563, doi:10.5194/hess-4-555-2000, 2000.

Liebe, H. J., Hufford, G. A., and Manabe, T.: A model for the complex permittivity of water at frequencies below $1 \mathrm{THz}$, Int. J. Infrared. Milli., 12, 659-675, 1991.

McLaughlin, D. and Coauthors: Short-wavelength technology and the potential for distributed networks of small radar systems, B. Am. Meteorol. Soc., 90, 1797-1817, 2009.

Otto, T., Figueras i Ventura, J., and Russchenberg, H. W. J.: Dropsize distribution retrieval of precipitation using a high-resolution polarimetric X-band weather radar, in: Proceedings of the 8th International Symposium of tropospheric profiling, Delft, The Netherlands, ISBN 978-90-6960-233-2, edited by: Apituley, A., Russchenberg, H. W. J., and Monna, W. A. A., 2009.

Testud, J., Le Bouar, E., Obligis, E., and Ali-Mehenni, M.: The Rain Profiling Algorithm Applied to Polarimetric Weather Radar, J. Atmos. Oceanic Technol., 17, 332-356, 2000. 